\title{
Socia/ Values for Ecosystem Services (SoIVES)—Using GIS to Include Social Values Information in Ecosystem Services Assessments
}

\section{Background}

Ecosystem services can be defined in various ways; simply put, they are the benefits provided by nature, which contribute to human well-being. These benefits can range from tangible products such as food and fresh water to cultural services such as recreation and esthetics. As the use of these benefits continues to increase, additional pressures are placed on the natural ecosystems providing them. This makes it all the more important when assessing possible tradeoffs among ecosystem services to consider the human attitudes and preferences that express underlying social values associated with their benefits. While some of these values can be accounted for through economic markets, other values can be more difficult to quantify, and attaching dollar amounts to them may not be very useful in all cases. Regardless of the processes or units used for quantifying such values, the ability to map them across the landscape and relate them to the ecosystem services to which they are attributed is necessary for effective assessments.

To address some of the needs associated with quantifying and mapping social values for inclusion in ecosystem services assessments, scientists at the Rocky Mountain Geographic Science Center (RMGSC), in collaboration with Colorado State University, have developed a public domain tool, Social Values for Ecosystem Services (SolVES). SolVES is a geographic information system (GIS) application designed to assess, map, and quantify social values for ecosystem services. SolVES calculates and maps a 10-point Value Index representing the relative perceived social values of ecosystem services such as recreation and biodiversity for various groups of ecosystem stakeholders. These groups are distinguished according to their attitudes and preferences regarding ecosystem uses such as motorized recreation or oil and gas drilling. Applying statistical techniques to SolVES output can identify and model relationships between social values and physical characteristics of the underlying landscape. These relationships can then be used to generate predicted Value Index maps for areas where survey data are not available.

\section{The SoIVES Application}

SolVES was developed as a custom toolbar for the Environmental Systems Research Institute's (ESRI@) $)^{1}$ ArcGIS software. It uses spatial and nonspatial responses from public attitude and preference surveys along with data layers representing attributes of the physical environment such as elevation and distance to roads. These data serve as inputs for three models: the Ecosystem Services Social Values Model, the Value Mapping Model, and the Value Transfer Mapping Model (fig. 1).

\section{SoIVES Overview}

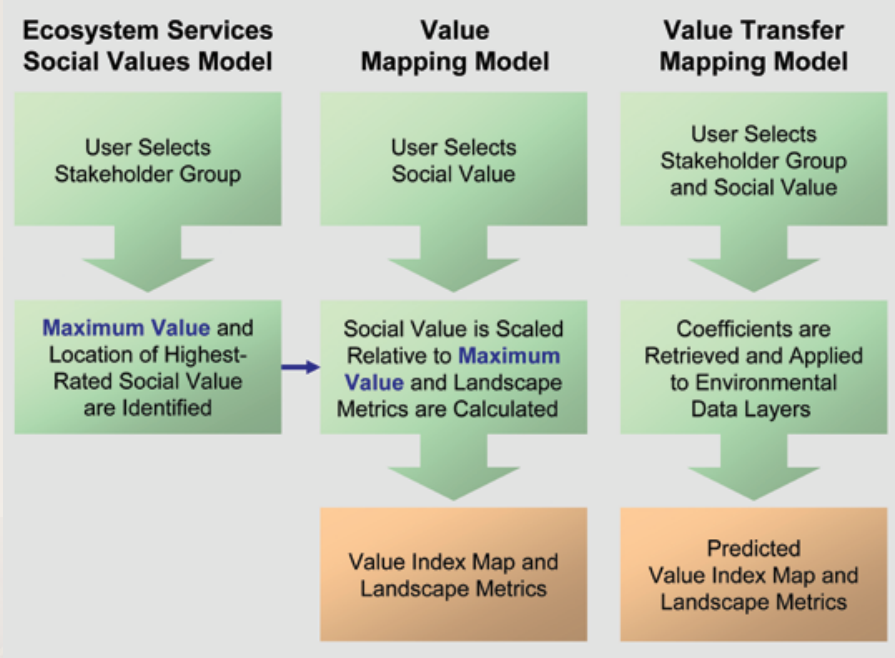

Figure 1. Generalized process flow of the SoIVES application's Ecosystem Services Social Values Model, Value Mapping Model, and Value Transfer Mapping Model.

Based on user inputs, the Ecosystem Services Social Values Model identifies the highest-rated social value for a specific stakeholder group as well as the location where it attains its maximum value. The Value Mapping Model then accepts the user's request for a specific social value, scales it relative to the maximum value previously identified by the Ecosystem Services Social Values. Model, and produces a Value Index map for the requested social value. The Value Mapping Model also uses the Value Index map to calculate a series of landscape metrics such as average distance to water and dominant land cover. In areas where survey data are not available, statistically derived coefficients calculated using output from the Ecosystem Services Social Values Model and the Value Mapping Model can be applied to their corresponding environmental data layers by the Value Transfer Mapping Model to generate predicted Value Index maps (fig. 2). 


\section{Future Research and Development}

The RMGSC is actively pursuing opportunities to work with land and resource managers as well as other researchers to apply SolVES to specific ecosystem management problems in a variety of physical and social contexts ranging from forest and rangeland to coastal and marine. An important research priority involves the investigation of effective methods for representing the Value Index in dollar terms to permit incorporation of SolVES results into comprehensive ecosystem assessment and valuation projects. These research efforts will support the continued development of more robust versions of SolVES that will facilitate the ability of managers, decisionmakers, and researchers to assess relative tradeoffs among ecosystem services and to communicate with diverse groups of stakeholders.

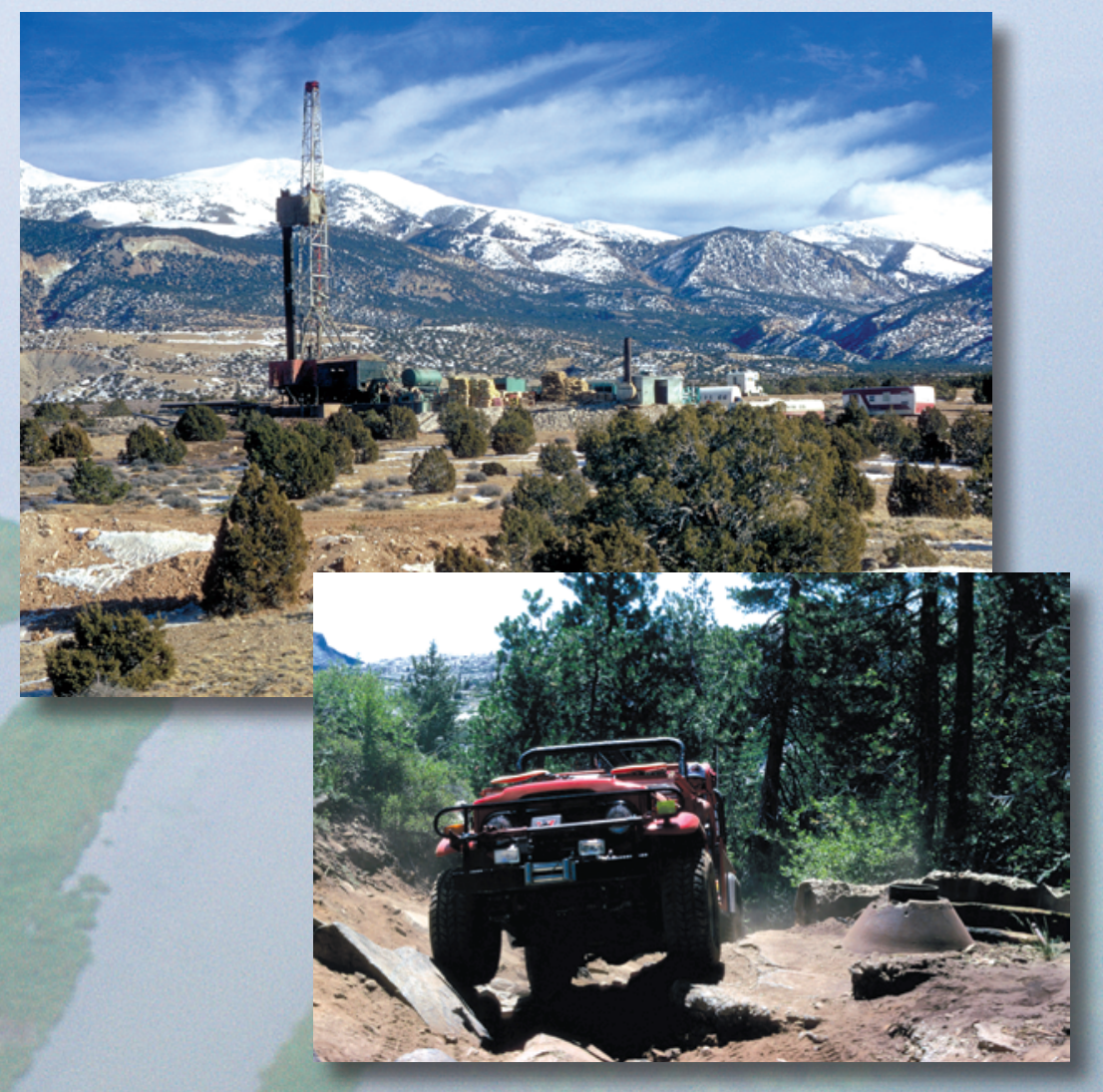

\section{SolVES Website Resources}

The SolVES Web site, located at http://solves.cr.usgs.gov, contains links to a downloadable file (SolVES.zip) containing the SolVES tool and sample data as well as to the following documentation:

- Social Values for Ecosystem Services (SolVES): A GIS Application for Assessing, Mapping, and Quantifying the Social Values of Ecosystem Services-Documentation and User Manual, Version 1.0 (Open-File Report 2010-1219). http://pubs.usgs.gov/of/2010/1219/pdf/OF10-1219.pdf

- SolVES Version 1.0 Quick Start Tutorial http://solves.cr.usgs.gov/downloads/SolVES_Quick_Start_Tutorial.pdf

- Journal article describing SolVES development and case study in press with Applied Geography (2010), doi:10.1016/j.apgeog.2010.08.002 http://solves.cr.usgs.gov/downloads/SolVES_GIS_App_Geog_preprint.pdf

- Sample public attitude and preference survey document http://solves.cr.usgs.gov/downloads/PSI_Final_Survey.pdf



Figure 2. Sample output from the Value Transfer Mapping Model representing recreation value for stakeholders who favor or strongly favor motorized recreation within the Pike and San Isabel National Forests in Colorado.

\section{For Additional Information}

To learn more about the development and application of SolVES, please contact:

\section{Ben Sherrouse}

Phone: 303-202-4072

bcsherrouse@usgs.gov

Darius Semmens

Phone: 303-578-6966

dsemmens@usgs.gov

U.S. Geological Survey

Rocky Mountain Geographic Science Center Denver Federal Center, Bldg. 810

P.O. Box 25046, Mail Stop 516

Denver, CO 80225

Fax: 303-202-4354 